groups $(4.88 \%$ among elementary, $2.16 \%$ secondary and $2.49 \%$ graduate students, $\mathrm{p}<0.001)$.

Conclusion Our results showed a high prevalence of selfreported syphilis in young adults of Brazil with significant difference between education level. Income and skin color/race were not associated with syphilis showing a disseminate outbreak. These data reinforce the importance of implementing strategies to combat this disease in young adults, especially among those with low educational level.

Disclosure No significant relationships.

\section{P733 EVALUATING THE USE OF RAPID SYPHILIS TESTING AMONG PATIENTS IN A SEXUALLY TRANSMITTED INFECTIONS CLINIC IN LILONGWE, MALAWI}

\begin{abstract}
${ }^{1} J a n e$ Chen*, ${ }^{2}$ Mitch Matoga, ${ }^{2}$ Shiraz Khan, ${ }^{2}$ Edward Jere, ${ }^{2}$ Cecilia Massa, ${ }^{2}$ Beatrice Ndalama, ${ }^{3}$ Arlene Seña, ${ }^{4}$ Kathryn Lancaster, ${ }^{3}$ Mina Hosseinipour, ${ }^{3}$ Myron Cohen, ${ }^{4}$ William Miller, ${ }^{3}$ Irving Hoffman. ' University of North Carolina at Chapel Hill, Epidemiology, Chapel Hill, USA; ${ }^{2}$ UNC Project Malawi, Lilongwe, Malawi; ${ }^{3}$ University of North Carolina at Chapel Hill, Division of Infectious Diseases, Chapel Hill, USA; ${ }^{4}$ The Ohio State University, Division of Epidemiology, Columbus, USA
\end{abstract}

10.1136/sextrans-2019-sti.793

Background Limited-resource countries, such as Malawi, rely largely on the syndromic diagnosis of genital ulcer disease (GUD) to detect and treat syphilis. However, rapid treponemal tests are available for point-of-care testing and offer inexpensive syphilis serology assessments, though they cannot differentiate between untreated and previously treated syphilis as a stand-alone test. We assessed syphilis seroprevalence in the sexually transmitted infections (STI) clinic at Bwaila District Hospital in Lilongwe, Malawi, in August 2017.

Methods Rapid syphilis testing (RST), with the Alere Determi$\mathrm{ne}^{\mathrm{TM}}$ Syphilis TP test or SD Bioline 3.0 Syphilis test, was offered in conjunction with standard opt-out HIV rapid testing. Anyone who tested RST positive was treated with three weekly doses of benzathine penicillin 2.4 MU IM, per Malawian standard of care. Per routine protocol, all patients also underwent a genital examination where GUD was diagnosed as the presence of one or more genital ulcers. We calculated syphilis seroprevalence, and used exact statistics to test for differences in proportions $(\alpha=0.05)$.

Results 848 patients had an RST, HIV test, and a genital exam, with $73(9 \%)$ testing positive by RST. Among the 82 patients (10\%) diagnosed with GUD, 26\% (95\% CI: 17\%$36 \%$ ) had a positive RST, compared to $7 \%$ (95\% CI: $5 \%-$ $9 \%)$ of patients without GUD $(\mathrm{p}<0.0001)$. Of the 89 patients $(10 \%)$ who tested newly positive for HIV, 19\% (95\% CI: 12\%-29\%) had a positive RST, compared to $7 \%$ (95\% CI: $6 \%-9 \%)$ among those who were HIV negative $(p=0.0009)$. Of the 73 patients who screened positive by RST, 71\% (95\% CI: $59 \%-81 \%$ ) did not have GUD.

Conclusion Syphilis serology was more prevalent among patients who had GUD and who were HIV-infected. Syndromic diagnosis of GUD may not be sufficient to identify patients who require syphilis treatment. However, accurate staging is critical for appropriate treatment, and concerns surrounding over treating previously treated cases should be addressed.

Disclosure No significant relationships.
P734 WHAT DO GUYS KNOW ABOUT SYPHILIS ANYWAYS?

${ }^{1}$ Dionne Gesink*, ${ }^{2} J a m e s$ Connell, 'Lauren Kimura. 'University of Toronto, Dalla Lana School of Public Health, Toronto, Canada; ${ }^{2}$ University of British Columbia, School of Population and Public Health, Vancouver, Canada

\subsection{6/sextrans-2019-sti.794}

Background Syphilis rates among gay, bisexual, and other men who have sex with men (gbMSM) have increased in Toronto, Canada, since the early 2000s. Half of syphilis cases are coinfected with HIV. Enhanced sexual health resources in the downtown neighbourhoods with elevated syphilis rates (core area) have not brought syphilis transmission under control. Our objective was to explore gbMSM attitudes, beliefs and knowledge of syphilis to inform syphilis intervention strategies.

Methods In-depth interviews were conducted with 31 gbMSM who lived, worked, or socialized in Toronto, Canada, in June and July of 2016. Interviews ran 60 to 90 minutes and had three sections. This analysis uses data from the third section, which explored what participants knew about syphilis and the syphilis epidemic in Toronto. Theoretical saturation for this analysis was reached before interviews were completed. Thematic analysis was used to analyze interviews. Transcripts were read and re-read, then coded. Codes were compared across participants then grouped into categories; categories were grouped into themes.

Results Participants knew little about syphilis and did not realize syphilis was epidemic in Toronto. Syphilis was perceived as a curable inconvenience and dismissed, while HIV was perceived as life-changing. Participants did know something about HIV and some STIs other than syphilis. Participants who lived through the 80's and 90's identified anal sex as a high-risk sexual activity and perceived sex as potentially lethal; consequently, sex was loaded with stigma, burden and shame. In backlash, some participants described a new social expectation that gay men should have a lot of good sex, where good sex is sex without a condom, and having good sex is a greater concern than acquiring an STI that can be cured or lived with.

Conclusion Dismissive attitudes towards syphilis could explain why syphilis epidemics persist. Interventions shifting gbMSM relationship with sex and STI testing should be explored.

Disclosure No significant relationships.

\section{P735 KNOWLEDGE AND ATTITUDES AROUND SYPHILIS AND SYPHILIS PRE-EXPOSURE PROPHYLAXIS AMONG MEN WHO HAVE SEX WITH MEN IN VANCOUVER}

${ }^{1}$ Ronita Nath*, ${ }^{2}$ Troy Grennan, ${ }^{2}$ Robin Parry, ${ }^{2}$ Fahmy Baharuddin, ${ }^{3}$ James Connell, ${ }^{2} J a s o n$ Wong, ${ }^{4}$ Daniel Grace. ${ }^{1}$ British Columbia Centre for Disease Control, Clinical Prevention, Vancouver, Canada; ${ }^{2} B C$ Centre for Disease Control, Clinical Prevention Services, Vancouver, Canada; ${ }^{3}$ British Columbia Centre for Disease Control, Clinical Prevention Services, Vancouver, Canada; ${ }^{4}$ University of Toronto, Dalla Lana School of Public Health, Toronto, Canada

\subsection{6/sextrans-2019-sti.795}

Background In British Columbia, Canada, syphilis is at recordhigh rates, with over $80 \%$ of cases in 2017 seen in gay, bisexual, and other men who have sex with men (GBM). The epidemic is of particular concern for those living with HIV, since 
syphilis may lead to more serious complications in this population. We sought to inductively explore syphilis-related knowledge, and attitudes around biomedical prevention options for syphilis in an age of HIV pre-exposure prophylaxis (PrEP), with the goal of informing effective strategies to address the syphilis epidemic.

Methods We conducted in-depth, one-on-one interviews with a heterogeneous sample of GBM in Vancouver, including men living with HIV and/or with a history of syphilis. Our interviews focused on participants' knowledge around syphilis and perceptions regarding syphilis PrEP. Interviews were audiorecorded, transcribed verbatim, and analyzed using Grounded Theory.

Results Twenty-five GBM were interviewed (64\% white; median age: 43 years). Four overarching themes emerged regarding men's views about syphilis. First, syphilis-related knowledge differed according to HIV and syphilis serostatus. Second, competing ideas emerged regarding men's concerns about syphilis. While our participants expressed concern about getting syphilis, they also described the importance of sexual intimacy and pleasure. Third, many participants said that syphilis was not perceived to be particularly alarming; preventing HIV infection remained a primary concern for many. Finally, although syphilis PrEP was appealing to some, participants were concerned about antibiotic resistance, cost, and side effects.

Conclusion Concern for syphilis appeared low among GBM. Our participants tended to organize their safer sex strategies around HIV, not syphilis. Although syphilis-related knowledge was relatively high among GBM living with HIV and those with a prior syphilis diagnosis, this knowledge did not appear to be associated with safer sexual practices, such as increased condom use. This work highlights the importance of examining other potential acceptable prevention solutions, such as syphilis PrEP.

Disclosure No significant relationships.

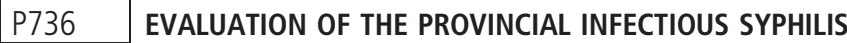 PARTNER NOTIFICATION PROGRAM IN BRITISH COLUMBIA, CANADA}

${ }^{1}$ Christine Lukac*, ${ }^{2}$ Theodora Consolacion, ${ }^{2}$ Venessa Ryan, ${ }^{2}$ Emma Cumming, ${ }^{2}$ Geoffrey Ford, ${ }^{1}$ Gina Ogilvie, ${ }^{2}$ Mark Gilbert, ${ }^{2}$ Troy Grennan, ${ }^{2} J a s o n$ Wong. ${ }^{1}$ University of British Columbia, Faculty of Medicine, Vancouver, Canada; ${ }^{2} B C$ Centre for Disease Control, Clinical Prevention Services, Vancouver, Canada

\subsection{6/sextrans-2019-sti.796}

Background Infectious syphilis partner notification $(\mathrm{PN})$ is centrally coordinated at the British Columbia (BC) Centre for Disease Control. Approaches include patient-initiated and provider-initiated $\mathrm{PN}$, and outcomes include the proportion of partners (1) notified of possible exposure to syphilis, (2) tested and/or treated, and (3) diagnosed. Among gay, bisexual and other men who have sex with men (gbMSM) who have the greatest burden of syphilis, we evaluated PN outcomes between patient-initiated and provider-initiated PN.

Methods All infectious syphilis diagnoses in 2016 in BC were included. Syphilis re-diagnosis was defined as a syphilis diagnosis in 2016 with at least one additional diagnosis in 20062016, while first-diagnosis was defined as a syphilis diagnosis in 2016 only. PN outcomes were calculated along a cascadeof-care framework, where the numerator is the denominator of the subsequent indicator. Chi-square tests compared PN outcomes of patient-initiated versus provider-initiated PN, within strata of gbMSM first-diagnosed and re-diagnosed.

Results Of the 759 infectious syphilis cases in BC in 2016, 648 (85\%) were among gbMSM, among whom 474 (73\%) were first-diagnoses and 174 (27\%) were re-diagnoses. A significantly greater proportion of gbMSM first-diagnosed chose patient-initiated PN compared to gbMSM re-diagnosed $(62 \%$ vs $42 \% ; \mathrm{P}<0.01)$. Among gbMSM first-diagnosed, patient-initiated $\mathrm{PN}$ resulted in a greater proportion of partners notified compared to provider-initiated PN (177/199; 89\% vs 426/603; $70 \% ; \mathrm{P}<0.001)$. There was no difference in the proportion of partners tested and/or treated, $(156 / 177 ; 88 \%$ vs $380 / 426$; $89 \% ; \mathrm{P} \geq 0.05)$, and diagnosed (24/156 15\% vs 51/380 13\%; $\mathrm{P} \geq 0.05)$. A similar trend in $\mathrm{PN}$ outcomes was observed among partners of gbMSM re-diagnosed.

Conclusion Patient-initiated and provider-initiated PN had similar outcomes among partners of both gbMSM first-diagnosed and re-diagnosed. However, gbMSM first-diagnosed were more likely to choose to notify their own partners. These findings demonstrate that patient-initiated $\mathrm{PN}$ have similar outcomes to provider-initiated $\mathrm{PN}$ and can increase the overall capacity for PN.

Disclosure No significant relationships.

\section{P737 EVALUATING SYPHILIS PARTNER NOTIFICATION OUTCOMES IN SEVEN JURISDICTIONS}

${ }^{1}$ Anna Cope*, ${ }^{2}$ James Matthias, ${ }^{3}$ Mohammad Rahman, ${ }^{4}$ Jill Diesel, ${ }^{5}$ River Pugsley, ${ }^{6}$ Julia Schillinger, ${ }^{7}$ Rilene $\mathrm{Ng},{ }^{8}$ Ellen Klingler, ${ }^{9}$ Victoria Mobley, ${ }^{9}$ Erika Samoff, ${ }^{10}$ Kyle Bernstein, ${ }^{11}$ Thomas Peterman. ${ }^{1}$ Centers for Disease Control and Prevention, Division of STD Prevention, Raleigh, USA; ${ }^{2}$ Centers for Disease Control and Prevention, Division of STD Prevention, Tallahassee, USA; ${ }^{3}$ Centers for Disease Control and Prevention, Division of STD Prevention, New Orleans, USA; ${ }^{4} C D C$, MDHHS, NCHHSTP, DSTDP, STD Section, Detroit, USA; ${ }^{5}$ Centers for Disease Control and Prevention, Division of STD Prevention, Richmond, USA; ${ }^{6}$ Centers for Disease Control and Prevention, Division of STD Prevention, New York City, USA; ${ }^{7}$ Centers for Disease Control and Prevention, Division of STD Prevention, San Francisco, USA; ${ }^{8}$ New York City Department of Health and Mental Hygiene, New York City, USA; ${ }^{9}$ North Carolina Division of Public Health, Communicable Disease Branch, Raleigh, USA; ${ }^{10}$ Centers for Disease Control and Prevention, Atlanta, USA; ${ }^{11}$ Centers for Disease Control and Prevention, Division of STD Prevention, Atlanta, USA

10.1136/sextrans-2019-sti.797

Background The effectiveness of partner notification services (PNS) for limiting syphilis transmission relies on the ability of disease intervention specialists (DIS) to find and assure treatment of partners. We measured estimates of partners found and treated due to PNS in seven jurisdictions.

Methods We reviewed early syphilis cases (primary, secondary, early latent) reported during 2015-2017 in seven jurisdictions in the United States (Florida, Louisiana, Michigan, North Carolina, Virginia, New York City, and San Francisco). We measured the numbers of: early syphilis (index) cases interviewed by DIS, (sex) partners reported (primary cases: $\leq 3$ months; secondary cases: $\leq 6$ months; early latent cases: $\leq 1$ year), partners with enough locating information to begin PNS, partners treated prophylactically, and infected partners brought in for treatment resulting from PNS. We considered partners to be brought to treatment by PNS if: 1) a DIS-assigned disposition code indicated "brought to treatment" or 2) the partner was treated 0-90 days after the index case was interviewed.

Results DIS interviewed 23,428 index patients with early syphilis (range among jurisdictions 1,106-9,388), representing $78.9 \%$ of reported cases $(50.1 \%-99.5 \%)$. Of those interviewed, $18,482(78.9 \%)$ reported 78,960 partners, of whom 